\title{
Trigonometric Function Periodic Wave Solutions and Their Limit Forms for the KdV-Like and the PC-Like Equations
}

\author{
Liu Zhengrong, Jiang Tianpei, Qin Peng, and Xu Qinfeng \\ Department of Mathematics, South China University of Technology, Guangzhou 510640, China \\ Correspondence should be addressed to Liu Zhengrong, liuzhr@scut.edu.cn \\ Received 15 February 2011; Accepted 26 May 2011 \\ Academic Editor: Ming Li
}

Copyright (c) 2011 Liu Zhengrong et al. This is an open access article distributed under the Creative Commons Attribution License, which permits unrestricted use, distribution, and reproduction in any medium, provided the original work is properly cited.

We use the bifurcation method of dynamical systems to study the periodic wave solutions and their limit forms for the KdV-like equation $u_{t}+a(1+b u) u u_{x}+u_{x x x}=0$, and PC-like equation $v_{t t}-v_{t t x x}-\left(a_{1} v+a_{2} v^{2}+a_{3} v^{3}\right)_{x x}=0$, respectively. Via some special phase orbits, we obtain some new explicit periodic wave solutions which are called trigonometric function periodic wave solutions because they are expressed in terms of trigonometric functions. We also show that the trigonometric function periodic wave solutions can be obtained from the limits of elliptic function periodic wave solutions. It is very interesting that the two equations have similar periodic wave solutions. Our work extend previous some results.

\section{Introduction}

Many authors have investigated the KdV-like equation

$$
u_{t}+a(1+b u) u u_{x}+u_{x x x}=0
$$

and the PC-like equation

$$
v_{t t}-v_{t t x x}-\left(a_{1} v+a_{2} v^{2}+a_{3} v^{3}\right)_{x x}=0
$$

For example, Dey $[1,2]$ studied the exact Himiltonian density and the conservation laws, and gave two kink solutions for (1.1). Zhang et al. [3, 4] gave some solitary wave solutions and singular wave solutions for (1.1) by using two different methods. Yu [5] got an exact kink soliton for (1.1) by using homogeneous balance method. Grimshaw et al. [6] studied the 
large-amplitude solitons for (1.1). Fan [7, 8] gave some bell-shaped soliton solutions, kinkshaped soliton, and Jacobi periodic solutions for (1.1) by using algebraic method. Tang et al. [9] investigated solitary waves and their bifurcations for (1.1) by employing bifurcation method of dynamical systems. Peng [10] used the modified mapping method to get some solitary wave solutions composed of hyperbolic functions, periodic wave solutions composed of Jacobi elliptic functions, and singular wave solution composed of triangle functions for (1.1). Chow et al. [11] described the interaction between a soliton and a breather for (1.1) by using the Hirota bilinear method. Kaya and Inan [12] studied solitary wave solutions for (1.1) by using Adomian decomposition method. Yomba [13] used Fan's subequation method to construct exact traveling wave solutions composed of hyperbolic functions or Jacobi elliptic functions for (1.1).

Zhang and Ma [14] gave some explicit solitary wave solutions composed of hyperbolic functions by using solving algebraic equations for (1.2). Li and Zhang [15] used bifurcation method of dynamical system to study the bifurcation of traveling wave solutions and construct solitary wave solutions for (1.2). Kaya [16] discussed the exact and numerical solitary wave solutions by using a decomposition method for (1.2). Rafei et al. [17] gave numerical solutions by using He's method for (1.2).

Recently, many authors have presented some useful methods to deal with the problems in equations, for instance [18-30].

In this paper, we use the bifurcation method mentioned above to study the periodic wave solutions for (1.1) and (1.2). Through some special phase orbits, we obtain new expressions of periodic wave solutions which are composed of trigonometric functions $\sin \xi$ or $\cos \xi$. These solutions are called trigonometric function periodic wave solutions. We also check the correctness by using the software Mathematica.

In Section 2, we will state our results for (1.1). In Section 3, we will state our results for (1.2). In Sections 4, and 5, we will give derivations for our main results. Some discussions and the orders for testing the correctness of the solutions will be given in Section 6 .

\section{Trigonometric Function Periodic Wave Solutions for (1.1)}

In this section, we state our main results for (1.1). In order to state these results conveniently, we give some preparations. For given constant $c \neq 0$, on $a-b$ plane we define some lines and regions as follows.

(1) When $c<0$, we define lines

$$
\begin{aligned}
& l_{1}: b=0, \\
& l_{2}: b=-\frac{a}{6 c}, \\
& l_{3}: b=-\frac{3 a}{16 c}, \\
& l_{4}: a=0,
\end{aligned}
$$

and regions $A_{i}(i=1-8)$, as Figure $1(\mathrm{a})$. 


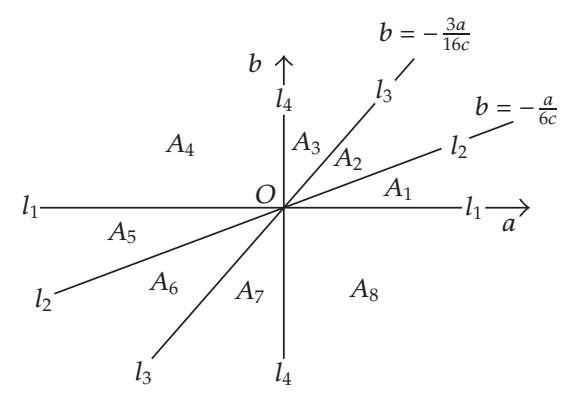

(a) $c<0$

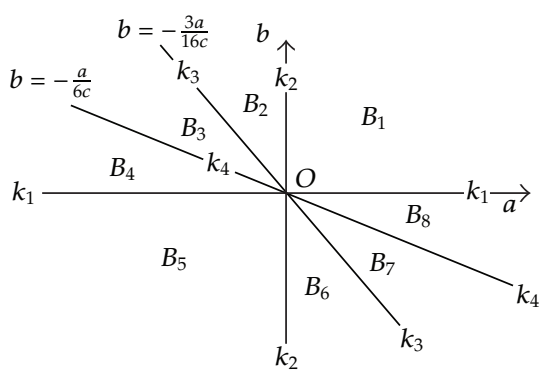

(b) $c>0$

Figure 1: The locations of the lines $l_{i}, k_{i}(i=1,2,3,4)$ and the regions $A_{j}, B_{j}(j=1,2, \ldots, 8)$ for given constant $c \neq 0$.

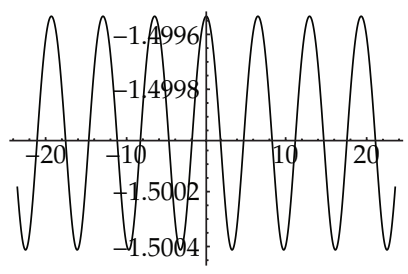

(a) for $b=0.66$

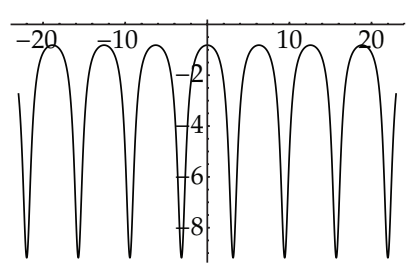

(b) for $b=0.2$

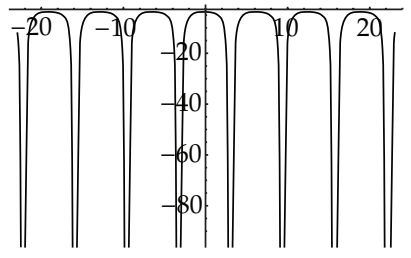

(c) for $b=0.00001$

Figure 2: The limiting precess of $u_{1}(\xi)$ when $c<0,(a, b) \in A_{1}$, and $(a, b)$ tends to the line $l_{1}$, where $a=4$ and $c=-1$.

(2) When $c>0$, we define lines

$$
\begin{aligned}
& k_{1}: b=0, \\
& k_{2}: a=0, \\
& k_{3}: b=-\frac{3 a}{16 c}, \\
& k_{4}: b=-\frac{a}{6 c},
\end{aligned}
$$

and regions $B_{i}(i=1-8)$, as Figure $1(b)$.

Using the lines and regions in Figure 1, we narrate our results as follows.

Proposition 2.1. For arbitrary given constant $c \neq 0$, let

$$
\xi=x-c t .
$$

Then, (1.1) has the following periodic wave solutions. 


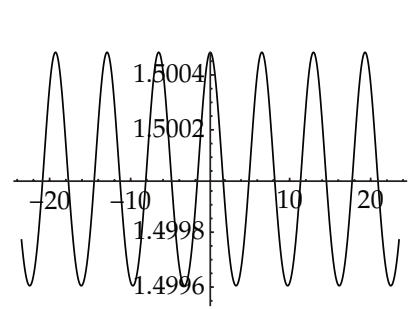

(a) for $b=-0.66$

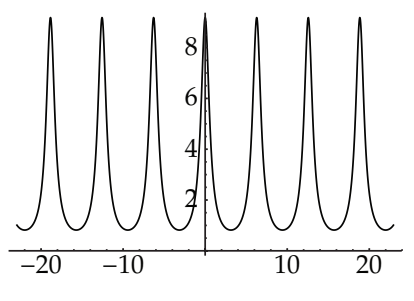

(b) for $b=-0.2$

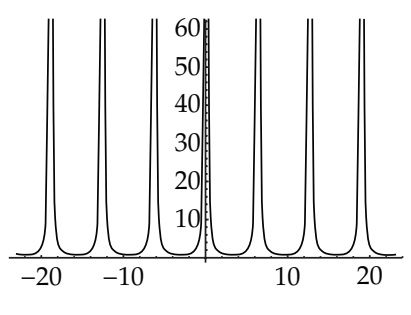

(c) for $b=-0.00001$

Figure 3: The limiting precess of $u_{1}(\xi)$ when $c<0,(a, b) \in A_{5}$, and $(a, b)$ tends to the line $l_{1}$, where $a=-4$ and $c=-1$.

(1) When $c<0$ and $(a, b) \in A_{1}$ or $A_{5}$, the expression of the periodic wave solution is

$$
u_{1}(\xi)=\frac{6 c}{a+\sqrt{a(a+6 b c)} \cos (\sqrt{-c} \xi)}
$$

which has the following limit forms.

(i) When $c<0,(a, b) \in A_{1}$ and $(a, b)$ tends to the line $l_{1}, u_{1}(\xi)$ tends to the periodic blow-up solution

$$
u_{1}^{\circ}(\xi)=\frac{6 c}{a[1+\cos (\sqrt{-c} \xi)]}
$$

(see Figure 2).

(ii) When $c<0,(a, b) \in A_{5}$ and $(a, b)$ tends to the line $l_{1}, u_{1}(\xi)$ tends to the periodic blow-up solution

$$
u_{1}^{*}(\xi)=\frac{6 c}{a[1-\cos (\sqrt{-c} \xi)]}
$$

(see Figure 3).

(iii) When $c<0,(a, b) \in A_{1}$ or $A_{5}$, and $(a, b)$ tends to $l_{2}, u_{1}(\xi)$ tends to the trivial solution $u(\xi)=6 c / a$.

(2) When $c<0$ and $(a, b) \in A_{2}$, or when $c>0$ and $(a, b) \in B_{5}$, the expression of the periodic wave solution is

$$
u_{2}(\xi)=\frac{\alpha_{0} \cos \left(w_{0} \xi\right)+\beta_{0}}{p_{0} \cos \left(w_{0} \xi\right)+q_{0}}
$$


where

$$
\begin{gathered}
\Delta=3 a(3 a+16 b c), \\
\alpha_{0}=-\frac{(3 a+\sqrt{\Delta}) \sqrt{a(a-\sqrt{\Delta})}}{4 a^{2} b^{2}}, \\
\beta_{0}=-\frac{3 a+24 b c+\sqrt{\Delta}}{2 a b^{2}}, \\
p_{0}=\frac{\sqrt{a(a-\sqrt{\Delta})}}{a b}, \\
q_{0}=-\frac{1}{a b}(a+\sqrt{\Delta}) \\
w_{0}=\sqrt{\frac{3 a+16 b c+\sqrt{\Delta}}{8 b} .}
\end{gathered}
$$

The solution $u_{2}(\xi)$ has the following limit forms.

(i) When $c<0,(a, b) \in A_{2}$ and $(a, b)$ tends to $l_{3}$, the $u_{2}(\xi)$ tends to the peak-shaped solitary wave solution

$$
u_{2}^{\circ}(\xi)=\frac{4 c\left(3+2 c \xi^{2}\right)}{a\left(-9+2 c \xi^{2}\right)}
$$

(see Figure 4).

(ii) When $c<0,(a, b) \in A_{2}$ and $(a, b)$ tends to $l_{2}, u_{2}(\xi)$ tends to the trivial solution $u(\xi)=0$.

(iii) When $c>0,(a, b) \in B_{5}$ and $(a, b)$ tends to $k_{1}$, the $u_{2}(\xi)$ tends to the periodic blow-up solution

$$
u_{2}^{*}(\xi)=\frac{c\left(2 \sin ^{2}(\sqrt{c} \xi / 2)-3\right)}{a \sin ^{2}(\sqrt{c} \xi / 2)}
$$

(see Figure 5).

(3) When $c<0$ and $(a, b) \in A_{6}$, or when $c>0$ and $(a, b) \in B_{1}$, the expressions of the solution is

$$
u_{3}(\xi)=\frac{\alpha_{1} \cos \left(w_{1} \xi\right)+\beta_{1}}{p_{1} \cos \left(w_{1} \xi\right)+q_{1}}
$$




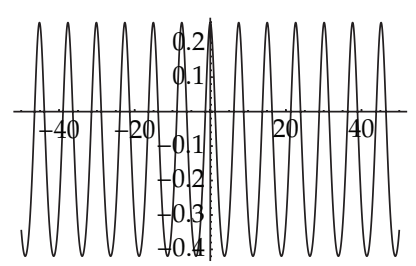

(a) for $b=0.7$

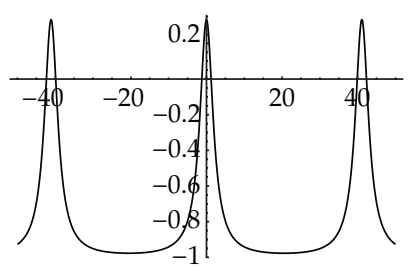

(b) for $b=0.7499$

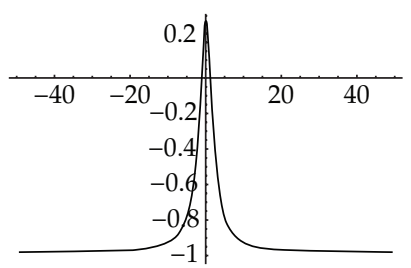

(c) for $b=0.7499999$

Figure 4: The limiting precess of $u_{2}(\xi)$ when $c<0,(a, b) \in A_{2}$, and $(a, b)$ tends to the line $l_{3}$, where $a=4$ and $c=-1$.

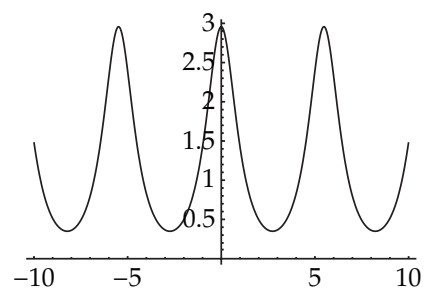

(a) for $b=-1$

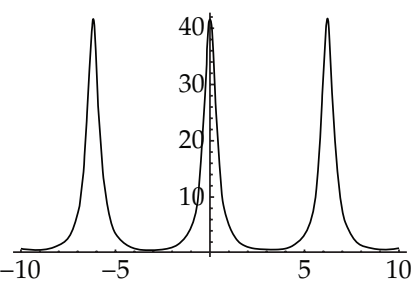

(b) for $b=-0.05$

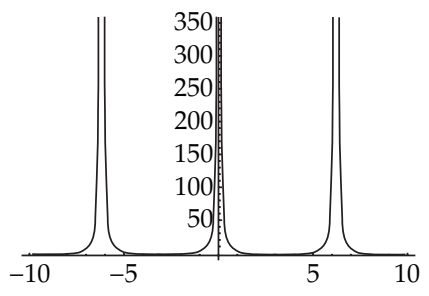

(c) for $b=-0.0001$

Figure 5: The limiting precess of $u_{2}(\xi)$ when $c>0,(a, b) \in B_{5}$, and $(a, b)$ tends to the line $k_{1}$, where $a=-2$ and $c=1$.

where

$$
\begin{gathered}
\alpha_{1}=\frac{(-3 a+\sqrt{\Delta}) \sqrt{a(a+\sqrt{\Delta})}}{4 a^{2} b^{2}}, \\
\beta_{1}=\frac{3 a+24 b c-\sqrt{\Delta}}{2 a b^{2}}, \\
p_{1}=\frac{\sqrt{a(a+\sqrt{\Delta})}}{a b}, \\
q_{1}=\frac{a-\sqrt{\Delta}}{a b}, \\
w_{1}=\sqrt{\frac{3 a+16 b c-\sqrt{\Delta}}{8 b} .}
\end{gathered}
$$

The solution $u_{3}(\xi)$ has the following limit forms.

(i) When $c<0,(a, b) \in A_{6}$ and $(a, b)$ tends to $l_{3}$, the $u_{3}(\xi)$ tends to the canyon-shaped solitary wave (see Figure 6) solution $u_{2}^{\circ}(\xi)$.

(ii) When $c<0,(a, b) \in A_{6}$ and $(a, b)$ tends to $l_{2}, u_{3}(\xi)$ tends to the trivial solution $u(\xi)=0$.

(iii) When $c>0,(a, b) \in B_{1}$ and $(a, b)$ tends to $k_{1}$, the $u_{3}(\xi)$ tends to the periodic blow-up wave solution $u_{1}^{*}(\xi)$ (see Figure 3 ). 


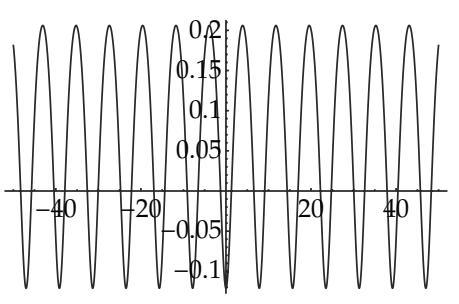

(a) for $b=-1.5875$

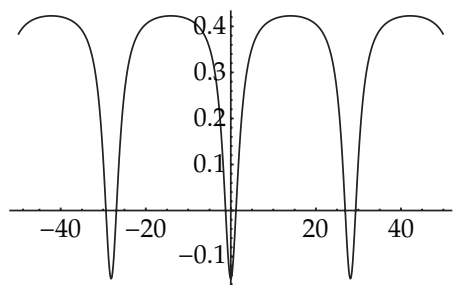

(b) for $b=-1.6865$

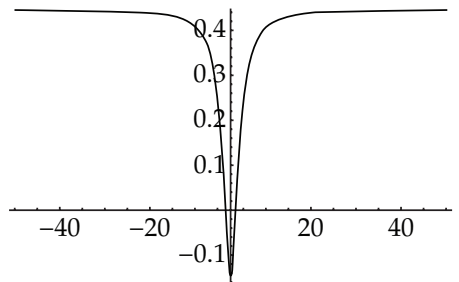

(c) for $b=-1.687499$

Figure 6: The limiting precess of $u_{3}(\xi)$ when $c<0,(a, b) \in A_{6}$, and $(a, b)$ tends to the line $l_{3}$, where $a=-9$ and $c=-1$.

Remark 2.2. Note that if $u=\varphi(\xi)$ is a solution of (1.1), then $u=\varphi(\xi+r)$ also is solution of (1.1), where $r$ is a arbitrary constant. According to this fact and the results listed in Proposition 2.1, the following nine functions also are periodic wave solutions of (1.1).

(1) When $c<0$ and $(a, b) \in A_{1}$ or $A_{5}$, the functions are

$$
\begin{aligned}
& u_{1}^{1}(\xi)=\frac{6 c}{a-\sqrt{a(a+6 b c)} \cos (\sqrt{-c} \xi)} \\
& u_{2}^{1}(\xi)=\frac{6 c}{a+\sqrt{a(a+6 b c)} \sin (\sqrt{-c} \xi)} \\
& u_{3}^{1}(\xi)=\frac{6 c}{a-\sqrt{a(a+6 b c)} \sin (\sqrt{-c} \xi)}
\end{aligned}
$$

(2) When $c<0$ and $(a, b) \in A_{2}$ or when $c>0$ and $(a, b) \in B_{5}$, the functions are

$$
\begin{aligned}
u_{1}^{2}(\xi) & =\frac{-\alpha_{0} \cos \left(w_{0} \xi\right)+\beta_{0}}{-p_{0} \cos \left(w_{0} \xi\right)+q_{0}}, \\
u_{2}^{2}(\xi) & =\frac{\alpha_{0} \sin \left(w_{0} \xi\right)+\beta_{0}}{p_{0} \sin \left(w_{0} \xi\right)+q_{0}}, \\
u_{3}^{2}(\xi) & =\frac{-\alpha_{0} \sin \left(w_{0} \xi\right)+\beta_{0}}{-p_{0} \sin \left(w_{0} \xi\right)+q_{0}} .
\end{aligned}
$$

(3) When $c<0$ and $(a, b) \in A_{6}$, or when $c>0$ and $(a, b) \in B_{1}$, the functions are

$$
\begin{aligned}
& u_{1}^{3}(\xi)=\frac{-\alpha_{1} \cos \left(w_{1} \xi\right)+\beta_{1}}{-p_{1} \cos \left(w_{1} \xi\right)+q_{1}}, \\
& u_{2}^{3}(\xi)=\frac{\alpha_{1} \sin \left(w_{1} \xi\right)+\beta_{1}}{p_{1} \sin \left(w_{1} \xi\right)+q_{1}}, \\
& u_{3}^{3}(\xi)=\frac{-\alpha_{1} \sin \left(w_{1} \xi\right)+\beta_{1}}{-p_{1} \sin \left(w_{1} \xi\right)+q_{1}} .
\end{aligned}
$$




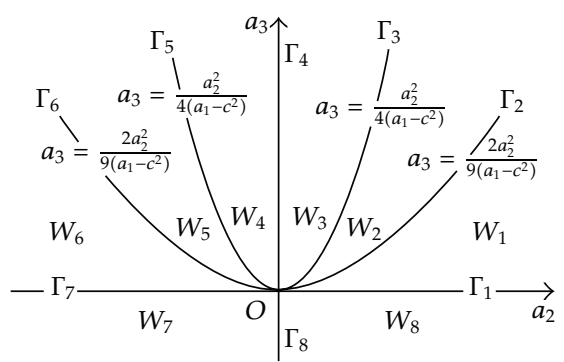

(a) $c^{2}<a_{1}$

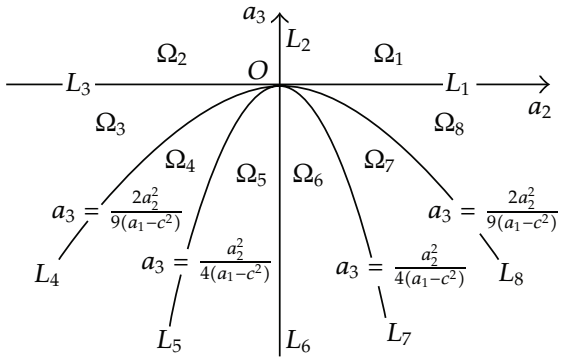

(b) $c^{2}>a_{1}$

Figure 7: The locations of the rays $\Gamma_{i}, L_{i}$ and the regions $W_{i}, \Omega_{i}(i=1,2, \ldots, 8)$ for given $a_{1}$ and $c$.

Remark 2.3. In the given parametric regions, the solutions $u_{i}(\xi), u_{i}^{1}(\xi), u_{i}^{2}(\xi), u_{i}^{3}(\xi)(i=1,2,3)$, and $u_{2}^{\circ}(\xi)$ are nonsingular. The solutions $u_{1}^{\circ}(\xi), u_{1}^{*}(\xi)$, and $u_{2}^{*}(\xi)$ are singular. The relationships of singular solutions and nonsingular solutions are displayed in the Proposition 2.1.

\section{Trigonometric Function Periodic Wave Solutions for (1.2)}

In this section, we state our main results for (1.2). For given $a_{1}$ and $c\left(a_{1} \neq c^{2}\right)$, on $a_{2}-a_{3}$ plane we define some rays and regions as follows.

(1) When $c^{2}<a_{1}$, we define curves

$$
\begin{aligned}
& \Gamma_{1}: a_{2}>0, \quad a_{3}=0, \\
& \Gamma_{2}: a_{2}>0, \quad a_{3}=\frac{2 a_{2}^{2}}{9\left(a_{1}-c^{2}\right)}, \\
& \Gamma_{3}: a_{2}>0, \quad a_{3}=\frac{a_{2}^{2}}{4\left(a_{1}-c^{2}\right)}, \\
& \Gamma_{4}: a_{2}=0, \quad a_{3}>0, \\
& \Gamma_{5}: a_{2}<0, \quad a_{3}=\frac{a_{2}^{2}}{4\left(a_{1}-c^{2}\right)}, \\
& \Gamma_{6}: a_{2}<0, \quad a_{3}=\frac{2 a_{2}^{2}}{9\left(a_{1}-c^{2}\right)}, \\
& \Gamma_{7}: a_{2}<0, \quad a_{3}=0, \\
& \Gamma_{8}: a_{2}=0, \quad a_{3}<0,
\end{aligned}
$$

and region $W_{i}$ as the domain surrounded by $\Gamma_{i}$ and $\Gamma_{i+1}(i=1-7), W_{8}$ as the domain surrounded by $\Gamma_{8}$ and $\Gamma_{1}$ (see Figure $7(a)$ ). 
Mathematical Problems in Engineering

(2) When $c^{2}>a_{1}$, we define curves

$$
\begin{aligned}
& L_{1}: a_{2}>0, \quad a_{3}=0, \\
& L_{2}: a_{2}=0, \quad a_{3}>0, \\
& L_{3}: a_{2}<0, \quad a_{3}=0, \\
& L_{4}: a_{2}<0, \quad a_{3}=\frac{2 a_{2}^{2}}{9\left(a_{1}-c^{2}\right)}, \\
& L_{5}: a_{2}<0, \quad a_{3}=\frac{a_{1}^{2}}{4\left(a_{1}-c^{2}\right)}, \\
& L_{6}: a_{2}=0, \quad a_{3}<0, \\
& L_{7}: a_{2}>0, \quad a_{3}=\frac{a_{2}^{2}}{4\left(a_{1}-c^{2}\right)}, \\
& L_{8}: a_{2}>0, \quad a_{3}=\frac{2 a_{2}^{2}}{9\left(a_{1}-c^{2}\right)},
\end{aligned}
$$

and region $\Omega_{i}$ as the domain surrounded by $L_{i}$ and $L_{i+1}(i=1-7), \Omega_{8}$ as the domain surrounded by $L_{8}$ and $L_{1}$ (see Figure $7(b)$ ).

Using the rays and regions above, we state our results as follows.

Proposition 3.1. For given parameter $a_{1}$ and constant $c$ satisfying $c^{2} \neq a_{1}$, let $\xi=x-c t$. Then, (1.2) has the following periodic wave solutions.

(1) When $c^{2}<a_{1}$ and $\left(a_{2}, a_{3}\right) \in W_{1}$ or $W_{6}$, the expression of the periodic wave solution is

$$
v_{1}(\xi)=\frac{R_{0}}{R_{1}+R_{2} \cos \left(R_{3} \xi\right)}
$$

where

$$
\begin{aligned}
& R_{0}=2\left(c^{2}-a_{1}\right), \\
& R_{1}=\frac{2 a_{2}}{3} \\
& R_{2}=\frac{1}{3} \sqrt{18 a_{3}\left(c^{2}-a_{1}\right)+4 a_{2}^{2}} \\
& R_{3}=\sqrt{\frac{a_{1}-c^{2}}{c^{2}}} .
\end{aligned}
$$

For $a_{2} \neq 0$, the periodic wave solution $v_{1}(\xi)$ has the following limit forms. 
(i) When $c^{2}<a_{1},\left(a_{2}, a_{3}\right) \in W_{1}$ and $\left(a_{2}, a_{3}\right)$ tends to the ray $\Gamma_{1}, v_{1}(\xi)$ tends to the periodic blow-up solution

$$
v_{1}^{\circ}(\xi)=\frac{3\left(c^{2}-a_{1}\right)}{a_{2}\left(1+\cos \left(\left(\sqrt{a_{1}-c^{2}} /|c|\right) \xi\right)\right)} .
$$

The limiting process is similar to that in Figure 2.

(ii) When $c^{2}<a_{1},\left(a_{2}, a_{3}\right) \in W_{6}$ and $\left(a_{2}, a_{3}\right)$ tends to the ray $\Gamma_{7}, v_{1}(\xi)$ tends to the periodic blow-up solution

$$
v_{1}^{*}(\xi)=\frac{3\left(c^{2}-a_{1}\right)}{a_{2}\left(1-\cos \left(\left(\sqrt{a_{1}-c^{2}} /|c|\right) \xi\right)\right)} .
$$

The limiting process is similar to that in Figure 3.

(iii) When $c^{2}<a_{1},\left(a_{2}, a_{3}\right) \in W_{1}$ and $\left(a_{2}, a_{3}\right)$ tends to the curve $\Gamma_{2}$, or $\left(a_{2}, a_{3}\right) \in W_{6}$ and $\left(a_{2}, a_{3}\right)$ tends to the curve $\Gamma_{6}, v_{1}(\xi)$ tends to the trivial solution $v(\xi)=3\left(c^{2}-a_{1}\right) / a_{2}$.

(2) When $c^{2}<a_{1}$ and $\left(a_{2}, a_{3}\right) \in W_{5}$, or when $c^{2}>a_{1}$ and $\left(a_{2}, a_{3}\right) \in \Omega_{1}$, the expression of the periodic wave solution is

$$
v_{2}(\xi)=S_{0}-\frac{2 S_{1}}{-S_{2}+S_{3} \cos \left(S_{4} \xi\right)}
$$

where

$$
\begin{aligned}
& S_{0}=\frac{-a_{2}+\sqrt{\omega}}{2 a_{3}}, \\
& S_{1}=\frac{-a_{2}^{2}+4 a_{3}\left(a_{1}-c^{2}\right)+a_{2} \sqrt{\omega}}{a_{3}^{2}}, \\
& S_{2}=\frac{2}{3 a_{3}}\left(-a_{2}+3 \sqrt{\omega}\right), \\
& S_{3}=\frac{2}{3 a_{3}} \sqrt{a_{2}\left(a_{2}+3 \sqrt{\omega}\right)}, \\
& S_{4}=\sqrt{-\frac{S_{1} a_{3}}{2 c^{2}}} \\
& \omega=a_{2}^{2}-4 a_{3}\left(a_{1}-c^{2}\right) .
\end{aligned}
$$

The periodic wave solution $v_{2}(\xi)$ has the following limit forms.

(i) When $c^{2}<a_{1},\left(a_{2}, a_{3}\right) \in W_{5}$, and $\left(a_{2}, a_{3}\right)$ tends to the curve $\Gamma_{6}, v_{2}(\xi)$ tends to the trivial solution $v(\xi)=0$. 
(ii) When $c^{2}<a_{1},\left(a_{2}, a_{3}\right) \in W_{5}$, and $\left(a_{2}, a_{3}\right)$ tends to the curve $\Gamma_{5}$, the $v_{2}(\xi)$ tends to the canyon-shaped solitary wave solution

$$
v_{2}^{\circ}(\xi)=\frac{2\left(a_{1}-c^{2}\right)\left[12 c^{2}-9 c^{2}-2\left(a_{1}-c^{2}\right) \xi^{2}\right]}{a_{2}\left[9 c^{2}+2\left(a_{1}-c^{2}\right) \xi^{2}\right]} .
$$

The limiting process is similar to that in Figure 6.

(iii) When $c^{2}>a_{1},\left(a_{2}, a_{3}\right) \in \Omega_{1}$, and $\left(a_{2}, a_{3}\right)$ tends to the ray $L_{1}, v_{2}(\xi)$ tends to the periodic blow-up wave solution

$$
v_{2}^{*}(\xi)=\frac{a_{1}-c^{2}}{2 a_{2}}\left[1+3 \tan ^{2}\left(\sqrt{\frac{c^{2}-a_{1}}{4 c^{2}} \xi}\right)\right] .
$$

The limiting process is similar to that in Figure 2.

(3) When $c^{2}<a_{1}$ and $\left(a_{2}, a_{3}\right) \in W_{2}$, or when $c^{2}>a_{1}$ and $\left(a_{2}, a_{3}\right) \in \Omega_{2}$, the expression of the periodic wave solution is

$$
v_{3}(\xi)=T_{0}+\frac{2 T_{1}}{-T_{2}+T_{3} \cos \left(T_{4} \xi\right)}
$$

where

$$
\begin{aligned}
& T_{0}=\frac{-a_{2}-\sqrt{\omega}}{2 a_{3}}, \\
& T_{1}=\frac{-a_{2}^{2}+4 a_{3}\left(a_{1}-c^{2}\right)-a_{2} \sqrt{\omega}}{a_{3}^{2}} \\
& T_{2}=\frac{2}{3 a_{3}}\left(a_{2}+3 \sqrt{\omega}\right) \\
& T_{3}=\frac{2}{3 a_{3}} \sqrt{a_{2}\left(a_{2}-3 \sqrt{\omega}\right)} \\
& T_{4}=\sqrt{-\frac{T_{1} a_{3}}{2 c^{2}}} .
\end{aligned}
$$

The periodic wave solution $v_{3}(\xi)$ has the following limit forms:

(i) When $c^{2}<a_{1},\left(a_{2}, a_{3}\right) \in W_{2}$, and $\left(a_{2}, a_{3}\right)$ tends to the curve $\Gamma_{2}, v_{3}(\xi)$ tends to the trivial solution $v(\xi)=0$.

(ii) When $c^{2}<a_{1},\left(a_{2}, a_{3}\right) \in W_{2}$, and $\left(a_{2}, a_{3}\right)$ tends to the curve $\Gamma_{3}$, the $v_{3}(\xi)$ tends to the peak-shaped solitary wave solution $v_{2}^{\circ}(\xi)$. The limiting process is similar to that in Figure 4.

(iii) When $c^{2}>a_{1},\left(a_{2}, a_{3}\right) \in \Omega_{2}$, and $\left(a_{2}, a_{3}\right)$ tends to the ray $L_{3}$, the $v_{3}(\xi)$ tends to the periodic blow-up wave solution $v_{2}^{*}(\xi)$. The limiting process is similar to that in Figure 5. 
Remark 3.2. Similar to the reason in Remark 2.2, the following nine functions also are periodic wave solutions of (1.2).

(1) When $c^{2}<a_{1}$ and $\left(a_{2}, a_{3}\right) \in W_{1}$ or $W_{6}$, the functions are

$$
\begin{aligned}
v_{1}^{1}(\xi) & =\frac{R_{0}}{R_{1}-R_{2} \cos \left(R_{3} \xi\right)}, \\
v_{2}^{1}(\xi) & =\frac{R_{0}}{R_{1}+R_{2} \sin \left(R_{3} \xi\right)}, \\
v_{3}^{1}(\xi) & =\frac{R_{0}}{R_{1}-R_{2} \sin \left(R_{3} \xi\right)} .
\end{aligned}
$$

(2) When $c^{2}<a_{1}$ and $\left(a_{2}, a_{3}\right) \in W_{5}$ or when $c^{2}>a_{1}$ and $\left(a_{2}, a_{3}\right) \in \Omega_{1}$, the functions are

$$
\begin{aligned}
& v_{1}^{2}(\xi)=S_{0}+\frac{2 S_{1}}{S_{2}+S_{3} \cos \left(S_{4} \xi\right)}, \\
& v_{2}^{2}(\xi)=S_{0}-\frac{2 S_{1}}{-S_{2}+S_{3} \sin \left(S_{4} \xi\right)} \\
& v_{3}^{2}(\xi)=S_{0}+\frac{2 S_{1}}{S_{2}+S_{3} \sin \left(S_{4} \xi\right)} .
\end{aligned}
$$

(3) When $c^{2}<a_{1}$ and $\left(a_{2}, a_{3}\right) \in W_{2}$ or when $c^{2}>a_{1}$ and $\left(a_{2}, a_{3}\right) \in \Omega_{2}$, the functions are

$$
\begin{aligned}
& v_{1}^{3}(\xi)=T_{0}-\frac{2 T_{1}}{T_{2}+T_{3} \cos \left(T_{4} \xi\right)}, \\
& v_{2}^{3}(\xi)=T_{0}+\frac{2 T_{1}}{-T_{2}+T_{3} \sin \left(T_{4} \xi\right)}, \\
& v_{3}^{3}(\xi)=T_{0}-\frac{2 T_{1}}{T_{2}+T_{3} \sin \left(T_{4} \xi\right)} .
\end{aligned}
$$

Remark 3.3. In the given regions, the solutions $v_{i}(\xi), v_{i}^{1}(\xi), v_{i}^{2}(\xi), v_{i}^{3}(\xi)(i=1,2,3)$, and $v_{2}^{\circ}(\xi)$ are nonsingular. The solutions $v_{1}^{\circ}(\xi), v_{1}^{*}(\xi)$, and $v_{2}^{*}(\xi)$ are singular. The relationships of nonsingular solutions and singular solutions are displayed in Proposition 3.1.

\section{The Derivation on Proposition 2.1}

In order to derive the Proposition 2.1, letting $c$ be a constant and substituting $u=\varphi(\xi)$ with $\xi=x-c t$ into (1.1), we have

$$
-c \varphi^{\prime}+a \varphi \varphi^{\prime}+a b \varphi^{2} \varphi^{\prime}+\varphi^{\prime \prime \prime}=0 .
$$


Integrating (4.1) once and letting the integral constant be zero, it follows that

$$
-c \varphi+\frac{a}{2} \varphi^{2}+\frac{a b}{3} \varphi^{3}+\varphi^{\prime \prime}=0
$$

Letting $\varphi^{\prime}=y$, yields the following planar system:

$$
\varphi^{\prime}=y, \quad y^{\prime}=c \varphi-\frac{a}{2}-\frac{a b}{3} \varphi^{3} .
$$

Obviously, system (4.3) has the first integral

$$
6 y^{2}-6 c \varphi^{2}+2 a \varphi^{3}+a b \varphi^{4}=h .
$$

Let

$$
\begin{aligned}
& \varphi_{1}=\frac{-3 a-\sqrt{\Delta}}{4 a b}, \\
& \varphi_{2}=\frac{-3 a+\sqrt{\Delta}}{4 a b},
\end{aligned}
$$

where $\Delta$ is defined in (2.8). Then, it is easy to see that system (4.3) has three singular points $\left(\varphi_{1}, 0\right),(0,0)$ and $\left(\varphi_{2}, 0\right)$ when $\Delta>0$, two singular points $((-3 / 4 b), 0)$ and $(0,0)$ when $\Delta=0$, unique singular point $(0,0)$ when $\Delta<0$.

Let $e_{i}$ and $f_{i}(i=1,2,3)$ be, respectively,

$$
\begin{aligned}
& e_{1}=\frac{-a-\sqrt{a^{2}+6 a b c}}{a b} \\
& f_{1}=\frac{-a+\sqrt{a^{2}+6 a b c}}{a b} \\
& e_{2}=\frac{1}{4 a b}(-a+\sqrt{\Delta}-2 \sqrt{a(a-\sqrt{\Delta})})
\end{aligned}
$$




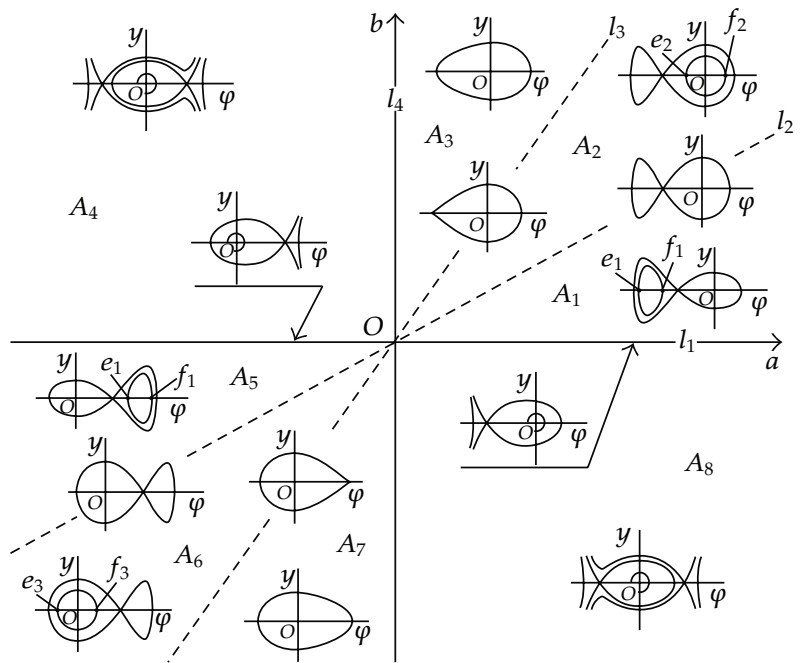

Figure 8: When $c<0$, the bifurcation phase portraits of system (4.3) and the locations of $e_{i}$ and $f_{i}(i=$ $1,2,3)$.

$$
\begin{aligned}
& f_{2}=\frac{1}{4 a b}(-a+\sqrt{\Delta}+2 \sqrt{a(a-\sqrt{\Delta})}), \\
& e_{3}=-\frac{1}{4 a b}(a+\sqrt{\Delta}+2 \sqrt{a(a+\sqrt{\Delta})}), \\
& f_{3}=-\frac{1}{4 a b}(a+\sqrt{\Delta}-2 \sqrt{a(a+\sqrt{\Delta})}) .
\end{aligned}
$$

Using the qualitative analysis of dynamical systems, we obtain the bifurcation phase portraits of system (4.3) and the locations of $e_{i}$ and $f_{i}(i=1,2,3)$ as Figures 8 and 9 .

It is easy to test that the closed orbit passing $\left(e_{i}, 0\right)$ passes $\left(f_{i}, 0\right)(i=1,2,3)$. Thus, using the phase portraits in Figures 8 and 9, we derive $u_{i}(\xi)(i=1,2,3)$ as follows.

(1) When $c<0$ and $(a, b) \in A_{1}$ or $A_{5}$, the closed orbit passing the points $\left(e_{1}, 0\right)$ and $\left(f_{1}, 0\right)$ has expression

$$
y= \pm \sqrt{\frac{a b}{6}} \varphi \sqrt{-e_{1} f_{1}+\left(e_{1}+f_{1}\right) \varphi-\varphi^{2}}, \quad \text { where } e_{1} \leq \varphi \leq f_{1}
$$

Substituting (4.7) into $\mathrm{d} \varphi / y=\mathrm{d} \xi$, we have

$$
\frac{\mathrm{d} \varphi}{\sqrt{-e_{1} f_{1}+\left(e_{1}+f_{1}\right) \varphi-\varphi^{2}}}=\sqrt{\frac{a b}{6}} \mathrm{~d} \xi
$$




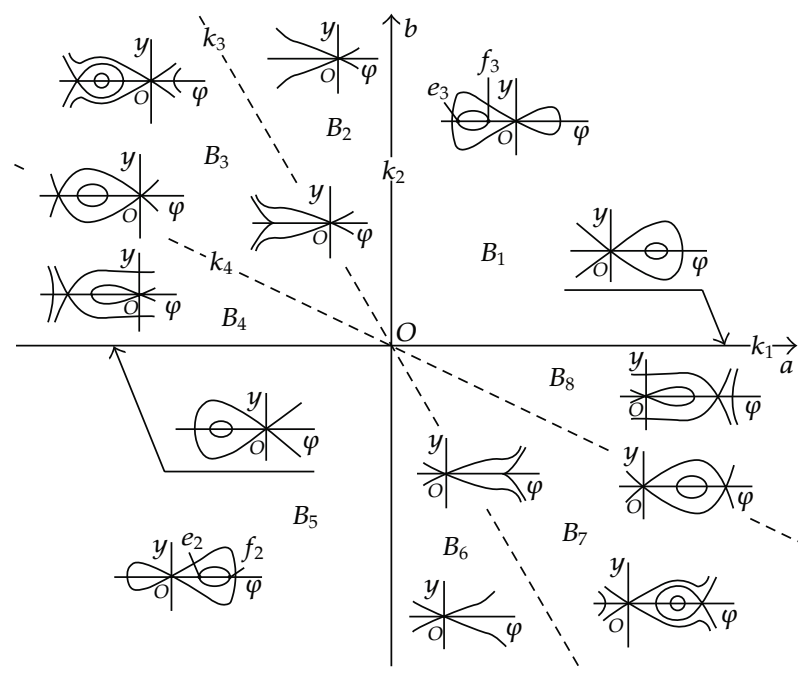

Figure 9: When $c>0$, the bifurcation phase portraits of system (4.3) and the locations of $e_{i}$ and $f_{i}(i=$ $1,2,3)$.

Integrating (4.8) along the closed orbit and noting that $u=\varphi(\xi)$, we obtain the solution $u_{1}(\xi)$ as (2.4).

(2) When $c<0$ and $(a, b) \in A_{2}$ or when $c>0$ and $(a, b) \in B_{5}$, the closed orbit passing the points $\left(e_{2}, 0\right)$ and $\left(f_{2}, 0\right)$ has expression

$$
y= \pm \sqrt{\frac{a b}{6}}\left(\varphi-\varphi_{1}\right) \sqrt{-e_{2} f_{2}+\left(e_{2}+f_{2}\right) \varphi-\varphi^{2}}, \quad \text { where } e_{2} \leq \varphi \leq f_{2}
$$

Substituting (4.9) into $\mathrm{d} \varphi / y=\mathrm{d} \xi$, we get

$$
\frac{\mathrm{d} \varphi}{\left(\varphi-\varphi_{1}\right) \sqrt{-e_{2} f_{2}+\left(e_{2}+f_{2}\right) \varphi-\varphi^{2}}}=\sqrt{\frac{a b}{6}} \mathrm{~d} \xi .
$$

Along the closed orbit integrating (4.10) and noting that $u=\varphi(\xi)$, we get the solution $u_{2}(\xi)$ as (2.7).

(3) When $c<0$ and $(a, b) \in A_{6}$ or when $c>0$ and $(a, b) \in B_{1}$, the closed orbit passing the points $\left(e_{3}, 0\right)$ and $\left(f_{3}, 0\right)$ has expression

$$
y= \pm \sqrt{\frac{a b}{6}}\left(\varphi_{2}-\varphi\right) \sqrt{-e_{3} f_{3}+\left(e_{3}+f_{3}\right) \varphi-\varphi^{2}}, \quad \text { where } e_{3} \leq \varphi \leq f_{3}
$$


Substituting (4.11) into $\mathrm{d} \varphi / y=\mathrm{d} \xi$, it follows that

$$
\frac{\mathrm{d} \varphi}{\left(\varphi_{2}-\varphi\right) \sqrt{-e_{3} f_{3}+\left(e_{3}+f_{3}\right) \varphi-\varphi^{2}}}=\sqrt{\frac{a b}{6}} \mathrm{~d} \xi .
$$

Similarly, along the closed orbit integrating (4.12), we obtain $u_{3}(\xi)$ as (2.16). From the expressions of these solutions, we get their limit forms. This completes the derivation on Proposition 2.1.

\section{The Derivation on Proposition 3.1}

In this section, we give derivation on Proposition 3.1. Let $v=\psi(\xi)$ with $\xi=x-c t$, where $c$ is a constant. Thus, (1.2) becomes

$$
c^{2} \psi^{\prime \prime}-c^{2} \psi^{\prime \prime \prime \prime}-\left(a_{1} \psi+a_{2} \psi^{2}+a_{3} \psi^{3}\right)^{\prime \prime}=0
$$

Integrating (5.1) twice and letting integral constant be zero, we get

$$
c^{2}\left(\psi-\psi^{\prime \prime}\right)=a_{1} \psi+a_{2} \psi^{2}+a_{3} \psi^{3}
$$

Letting $\psi^{\prime}=y$, we have the planar system

$$
\psi^{\prime}=y, \quad c^{2} y^{\prime}=\left(c^{2}-a_{1}\right) \psi-a_{2} \psi^{2}-a_{3} \psi^{3} .
$$

It is easy to see that system (5.3) has the first integral

$$
c^{2} y^{2}+\psi^{2}\left(\frac{a_{3}}{2} \psi^{2}+\frac{2 a_{2}}{3} \psi+a_{1}-c^{2}\right)=h,
$$

and three singular points $(0,0),\left(\psi_{1}, 0\right)$, and $\left(\psi_{2}, 0\right)$, where

$$
\begin{aligned}
& \psi_{1}=\frac{-a_{2}-\sqrt{\omega}}{2 a_{3}}, \\
& \psi_{2}=\frac{-a_{2}+\sqrt{\omega}}{2 a_{3}}
\end{aligned}
$$

and $\omega$ is defined in (3.9). 


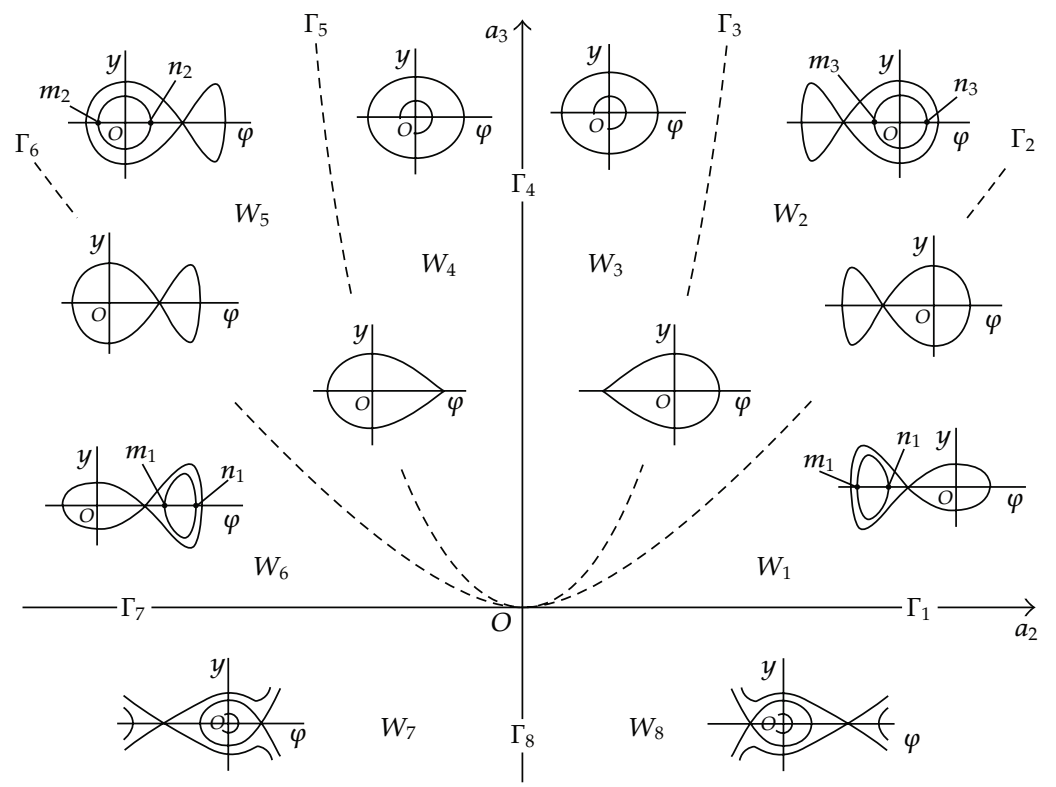

Figure 10: When $c^{2}<a_{1}$, the bifurcation phase portraits of system (5.3) and the locations of $m_{i}$ and $n_{i}(i=$ $1,2,3)$.

Let $m_{i}$ and $n_{i}(i=1,2,3)$ be, respectively,

$$
\begin{aligned}
& m_{1}=\frac{-2 a_{2}-\sqrt{2\left(a_{2}^{2}-9 a_{1} a_{3}+9 a_{3} c^{2}\right)}}{3 a_{3}}, \\
& n_{1}=\frac{-2 a_{2}+\sqrt{2\left(a_{2}^{2}-9 a_{1} a_{3}+9 a_{3} c^{2}\right)}}{3 a_{3}}, \\
& m_{2}=\frac{-a_{2}-3 \sqrt{\omega}-2 \sqrt{a_{2}\left(a_{2}+3 \sqrt{\omega}\right)}}{6 a_{3}}, \\
& n_{2}=\frac{-a_{2}-3 \sqrt{\omega}+2 \sqrt{a_{2}\left(a_{2}+3 \sqrt{\omega}\right)}}{6 a_{3}}, \\
& m_{3}=\frac{-a_{2}+3 \sqrt{\omega}-2 \sqrt{a_{2}\left(a_{2}-3 \sqrt{\omega}\right)}}{6 a_{3}} \\
& n_{3}=\frac{-a_{2}+3 \sqrt{\omega}+2 \sqrt{a_{2}\left(a_{2}-3 \sqrt{\omega}\right)}}{6 a_{3}} .
\end{aligned}
$$

Similarly, using the qualitative analysis of dynamical systems, we get the bifurcation phase portraits of system (5.3) and the locations of $m_{i}$ and $n_{i}(i=1,2,3)$ as Figures 10 and 11.

It is easy to test that the closed orbit passing $\left(m_{i}, 0\right)$ passes $\left(n_{i}, 0\right)(i=1,2,3)$. Thus, using the phase portraits in Figures 10 and 11, we derive $v_{i}(\xi)(i=1,2,3)$ as follows. 


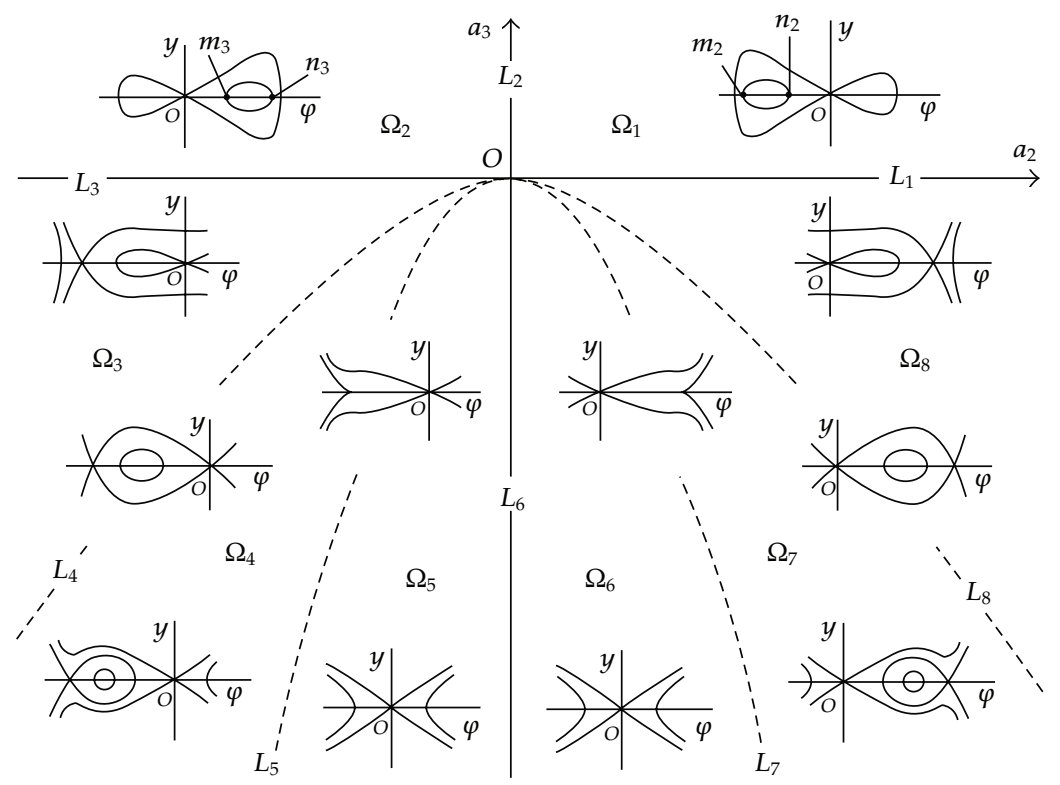

Figure 11: When $c^{2}>a_{1}$, the bifurcation phase portraits of system (5.3) and the locations of $m_{i}$ and $n_{i}(i=$ $1,2,3)$.

(1) When $c^{2}<a_{1}$ and $\left(a_{2}, a_{3}\right) \in W_{1}$ or $W_{6}$, the closed orbit passing the points $\left(m_{1}, 0\right)$ and $\left(n_{1}, 0\right)$ has expression

$$
y= \pm \sqrt{\frac{a_{3}}{2 c^{2}}} \psi \sqrt{-m_{1} n_{1}+\left(m_{1}+n_{1}\right) \psi-\psi^{2}}, \quad \text { where } m_{1} \leq \psi \leq n_{1}
$$

Substituting (5.7) into $\mathrm{d} \psi / y=\mathrm{d} \xi$, we have

$$
\frac{\mathrm{d} \psi}{\psi \sqrt{-m_{1} n_{1}+\left(m_{1}+n_{1}\right) \psi-\psi^{2}}}=\sqrt{\frac{a_{3}}{2 c^{2}}} \mathrm{~d} \xi .
$$

Integrating (5.8) along the closed orbit and noting that $v=\psi(\xi)$, we get the solution $v_{1}(\xi)$ as (3.3).

(2) When $c^{2}<a_{1}$ and $\left(a_{2}, a_{3}\right) \in W_{5}$, or when $c^{2}>a_{1}$ and $\left(a_{2}, a_{3}\right) \in \Omega_{1}$, the closed orbit passing the points $\left(m_{2}, 0\right)$ and $\left(n_{2}, 0\right)$ has expression

$$
y= \pm \sqrt{\frac{a_{3}}{2 c^{2}}}\left(\psi_{2}-\psi\right) \sqrt{-m_{2} n_{2}+\left(m_{2}+n_{2}\right) \psi-\psi^{2}}, \quad \text { where } m_{2} \leq \psi \leq n_{2}
$$


From $\mathrm{d} \psi / y=\mathrm{d} \xi$ and (5.9), it follows that

$$
\frac{\mathrm{d} \psi}{\left(\psi_{2}-\psi\right) \sqrt{-m_{2} n_{2}+\left(m_{2}+n_{2}\right) \psi-\psi^{2}}}=\sqrt{\frac{a_{3}}{2 c^{2}}} \mathrm{~d} \xi .
$$

Integrating (5.10) along the closed orbit, we get $v_{2}(\xi)$ as (3.7).

(3) When $c^{2}<a_{1}$ and $\left(a_{2}, a_{3}\right) \in W_{2}$, or when $c^{2}>a_{1}$ and $\left(a_{2}, a_{3}\right) \in \Omega_{2}$, the closed orbit passing the points $\left(m_{3}, 0\right)$ and $\left(n_{3}, 0\right)$ has expression

$$
y= \pm \sqrt{\frac{a_{3}}{2 c^{2}}}\left(\psi-\psi_{1}\right) \sqrt{-m_{3} n_{3}+\left(m_{3}+n_{3}\right) \psi-\psi^{2}}, \quad \text { where } m_{3} \leq \psi \leq n_{3} .
$$

Substituting (5.11) into $\mathrm{d} \psi / y=\mathrm{d} \xi$, we have

$$
\frac{\mathrm{d} \psi}{\left(\psi-\psi_{1}\right) \sqrt{-m_{3} n_{3}+\left(m_{3}+n_{3}\right) \psi-\psi^{2}}}=\sqrt{\frac{a_{3}}{2 c^{2}}} \mathrm{~d} \xi .
$$

Integrating (5.12) along the closed orbit, we obtain $v_{3}(\xi)$ as (3.12). From the expressions of these solutions, we get their limiting properties. This completes the derivation on Proposition 3.1.

\section{Discussions and Testing Orders}

In this paper, Using the special closed orbits, we have obtained trigonometric function periodic wave solutions for (1.1) and (1.2), respectively. Their limit forms have been given. From these expressions, an interesting phenomena has been seen, that is, (1.1) and (1.2) have similar periodic wave solutions. Our work has extended previous results on periodic wave solutions.

Now, we point out that the trigonometric function periodic wave solutions can be obtained from the limits of the elliplic function periodic wave solution. For given real number $\mu$, let

$$
\begin{aligned}
& \mu_{1}=\frac{1}{12 a b}\left(-4 a(2+b \mu)+\frac{4(1+\mathrm{i} \sqrt{3}) a F_{02}}{F}+2 \mathrm{i}(\mathrm{i}+\sqrt{3}) F\right), \\
& \mu_{2}=\frac{1}{12 a b}\left(-4 a(2+b \mu)+\frac{4(1-\mathrm{i} \sqrt{3}) a F_{02}}{F}-2 \mathrm{i}(\mathrm{i}+\sqrt{3}) F\right), \\
& \mu_{3}=\frac{1}{6 a b}\left(-2 a(2+b \mu)-\frac{4 a F_{02}}{F}+2 F\right),
\end{aligned}
$$




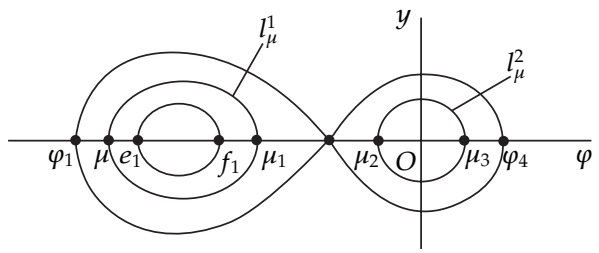

Figure 12: The locations of $l_{\mu}^{1}$ and $l_{\mu}^{2}$ when $c<0$ and $(a, b) \in A_{1}$.

where

$$
\begin{aligned}
F_{01} & =\left(8-6 b \mu+15 b^{2} \mu^{2}+10 b^{3} \mu^{3}\right), \\
F_{02} & =\left(-9 b c+a\left(-2+b \mu+b^{2} \mu^{2}\right)\right), \\
F_{03} & =\sqrt{a^{3}\left(8 F_{02}^{3}+a\left(-54 b c(-1+b \mu)+a F_{01}\right)^{2}\right)}, \\
F & =\left(54 a^{2} b c(-1+b \mu)-a^{3} F_{01}+F_{03}\right)^{1 / 3} .
\end{aligned}
$$

Assume that $c<0,(a, b) \in\left(A_{1}\right)$, and $\varphi_{1}<\mu<e_{1}$. It is easy to check that $\mu_{i}(i=1,2,3)$ are real and satisfy

$$
\mu<e_{1}<\varphi_{2}<f_{1}<\mu_{1}<\varphi_{3}<\mu_{2}<0<\mu_{3}<\varphi_{4} .
$$

There are two closed orbits $l_{\mu}^{1}$ and $l_{\mu}^{2}$ (see Figure 12). The closed orbit $l_{\mu}^{1}$ passes the points $(\mu, 0)$ and $\left(\mu_{1}, 0\right)$. The closed orbit $l_{\mu}^{2}$ passes the points $\left(\mu_{2}, 0\right)$ and $\left(\mu_{3}, 0\right)$.

On $\varphi-y$ plane, the expression of $l_{\mu}^{1}$ is

$$
y^{2}=\frac{a b}{6}\left(\mu_{3}-\varphi\right)\left(\mu_{2}-\varphi\right)\left(\mu_{1}-\varphi\right)(\varphi-\mu), \quad \text { where } \mu \leq \varphi \leq \mu_{1} .
$$

Substituting (6.4) into $\mathrm{d} \varphi / y=\mathrm{d} \xi$ and integrating it along $l_{\mu}^{1}$, we have

$$
g \operatorname{sn}^{-1}(\sin z, k)=\sqrt{\frac{a b}{6}}|\xi|
$$


where

$$
\begin{gathered}
g=\frac{2}{\sqrt{\left(\mu_{3}-\mu_{1}\right)\left(\mu_{2}-\mu\right)}}, \\
k=\sqrt{\frac{\left(\mu_{3}-\mu_{2}\right)\left(\mu_{1}-\mu\right)}{\left(\mu_{3}-\mu_{1}\right)\left(\mu_{2}-\mu\right)}}, \\
\sin z=\sqrt{\frac{\left(\mu_{3}-\mu_{1}\right)(\varphi-\mu)}{\left(\mu_{1}-\mu\right)\left(\mu_{3}-\varphi\right)}} .
\end{gathered}
$$

Solving (6.5) for $\varphi$ and noting that $u=\varphi(\xi)$, we obtain an elliptic function periodic wave solution

$$
u(\xi)=\frac{\mu\left(\mu_{3}-\mu_{1}\right)+\mu_{3}\left(\mu_{1}-\mu\right) \operatorname{sn}^{2}(\eta \xi, k)}{\mu_{3}-\mu_{1}+\left(\mu_{1}-\mu\right) \operatorname{sn}^{2}(\eta \xi, k)}
$$

where

$$
\eta=\sqrt{\frac{a b\left(\mu_{3}-\mu_{1}\right)\left(\mu_{2}-\mu\right)}{24}} .
$$

Letting $\mu \rightarrow e_{1}-0$, it follows that $\mu_{1} \rightarrow f_{1}, \mu_{2} \rightarrow 0, \mu_{3} \rightarrow 0, k \rightarrow 0, \eta \rightarrow$ $\sqrt{\left(a b e_{1} f_{1}\right) / 24}$ and $\operatorname{sn}^{2}(\eta \xi, k) \rightarrow \operatorname{sn}^{2}\left(\sqrt{\left(a b e_{1} f_{1} / 24\right)} \xi, 0\right)=\sin ^{2}\left(\sqrt{\left(a b e_{1} f_{1} / 24\right)} \xi\right)$.

Therefore, in (6.7) letting $\mu \rightarrow e_{1}-0$, we obtain the trigonometric function periodic wave solution

$$
\begin{aligned}
u(\xi) & =\frac{e_{1} f_{1}}{f_{1}+\left(e_{1}-f_{1}\right) \sin ^{2}\left(\sqrt{\left(a b e_{1} f_{1} / 24\right)} \xi\right)} \\
& =\frac{-6 c}{-a+\sqrt{a(a+6 b c)}-2 \sqrt{a(a+6 b c)} \sin ^{2}((\sqrt{|c|} / 2) \xi)} \\
& =\frac{6 c}{a-\sqrt{a(a+6 b c)} \cos (\sqrt{|c|} \xi)}=u_{1}^{1}(\xi) .
\end{aligned}
$$

Via Remark 2.2 and $u_{1}^{1}(\xi)$, further we get $u_{2}^{1}(\xi), u_{3}^{1}(\xi)$ and $u_{1}(\xi)$. Similarly, we can derive others trigonometric function periodic wave solutions.

We also have tested the correctness of each solution by using the software Mathematica. Here, we list two testing orders. Others testing orders are similar. 
(1) The orders for testing $u_{1}(\xi)$

$$
u=\frac{6 c}{a+\sqrt{a(a+6 b c)} \cos [\sqrt{-c}(x-c t)]}
$$

Simplify $[D[u, t]+a(1+b u) D[u, x] u+D[u,\{x, 3\}]]$.

(2) The orders for testing $v_{1}(\xi)$

$$
\begin{aligned}
\mathrm{R}_{0} & =2\left(-\mathrm{a}_{1}+\mathrm{c}^{2}\right), \\
\mathrm{R}_{1} & =\frac{2 \mathrm{a}_{2}}{3}, \\
\mathrm{R}_{2} & =\sqrt{2 \mathrm{a}_{3}\left(-\mathrm{a}_{1}+\mathrm{c}^{2}\right)+\frac{4 \mathrm{a}_{2}^{2}}{9}}, \\
\mathrm{R}_{3} & =\sqrt{\frac{\mathrm{a}_{1}-\mathrm{c}^{2}}{\mathrm{c}^{2}}}, \\
\mathrm{v} & =\frac{\mathrm{R}_{0}}{\mathrm{R}_{1}+\mathrm{R}_{2} \cos \left[\mathrm{R}_{3}(\mathrm{x}-\mathrm{ct})\right]}, \\
\mathrm{vtt} & =\mathrm{D}[\mathrm{v},\{\mathrm{t}, 2\}], \\
\mathrm{vttxx} & =\mathrm{D}[\mathrm{vtt},\{\mathrm{x}, 2\}]
\end{aligned}
$$

Simplify $\left[v t t-v t t x x-D\left[a_{1} v+a_{2} v^{2}+a_{3} v^{3},\{x, 2\}\right]\right]$.

\section{Acknowledgment}

Research is supported by the National Natural Science Foundation of China (no. 10871073) and the Research Expences of Central Universities for students.

\section{References}

[1] B. Dey, "Domain wall solutions of KdV-like equations with higher order nonlinearity," Journal of Physics A, vol. 19, no. 1, pp. L9-L12, 1986.

[2] B. Dey, "KdV like equations with domain wall solutions and their Hamiltonians," in Solitons, pp. 188-194, Springer, Berlin, Germany, 1988.

[3] J. F. Zhang, "New solitary wave solution of the combined $\mathrm{KdV}$ and $\mathrm{mKdV}$ equation," International Journal of Theoretical Physics, vol. 37, no. 5, pp. 1541-1546, 1998.

[4] J. F. Zhang, F. M. Wu, and J. Q. Shi, "Simple solition solution method for the combined KdV and mKdV equation," International Journal of Theoretical Physics, vol. 39, no. 6, pp. 1697-1702, 2000.

[5] J. Yu, "Exact solitary wave solutions to a combined $\mathrm{KdV}$ and mKdV equation," Mathematical Methods in the Applied Sciences, vol. 23, no. 18, pp. 1667-1670, 2000.

[6] R. Grimshaw, D. Pelinovsky, E. Pelinovsky, and A. Slunyaev, “Generation of large-amplitude solitons in the extended Korteweg-de Vries equation," Chaos, vol. 12, no. 4, pp. 1070-1076, 2002.

[7] E. G. Fan, "Multiple travelling wave solutions of nonlinear evolution equations using a unified algebraic method," Journal of Physics A, vol. 35, no. 32, pp. 6853-6872, 2002. 
[8] E. G. Fan, "Uniformly constructing a series of explicit exact solutions to nonlinear equations in mathematical physics," Chaos, Solitons and Fractals, vol. 16, no. 5, pp. 819-839, 2003.

[9] M. Y. Tang, R. Q. Wang, and Z. J. Jing, "Solitary waves and their bifurcations of KdV like equation with higher order nonlinearity," Science in China A, vol. 45, no. 10, pp. 1255-1267, 2002.

[10] Y.-Z. Peng, "New exact solutions to the combined KdV and mKdV equation," International Journal of Theoretical Physics, vol. 42, no. 4, pp. 863-868, 2003.

[11] K. W. Chow, R. H. J. Grimshaw, and E. Ding, "Interactions of breathers and solitons in the extended Korteweg-de Vries equation," Wave Motion, vol. 43, no. 2, pp. 158-166, 2005.

[12] D. Kaya and I. E. Inan, "A numerical application of the decomposition method for the combined KdV-MKdV equation," Applied Mathematics and Computation, vol. 168, no. 2, pp. 915-926, 2005.

[13] E. Yomba, "The extended Fan's sub-equation method and its application to KdV-MKdV, BKK and variant Boussinesq equations," Physics Letters A, vol. 336, no. 6, pp. 463-476, 2005.

[14] W. G. Zhang and W. X. Ma, "Explicit solitary-wave solutions to generalized Pochhammer-Chree equations," Applied Mathematics and Mechanics, vol. 20, no. 6, pp. 625-632, 1999.

[15] J. B. Li and L. J. Zhang, "Bifurcations of traveling wave solutions in generalized Pochhammer-Chree equation," Chaos, Solitons and Fractals, vol. 14, no. 4, pp. 581-593, 2002.

[16] D. Kaya, "The exact and numerical solitary-wave solutions for generalized modified Boussinesq equation," Physics Letters A, vol. 348, no. 3-6, pp. 244-250, 2006.

[17] M. Rafei, D. D. Ganji, H. R. M. Daniali, and H. Pashaei, "Application of homotopy perturbation method to the RLW and generalized modified Boussinesq equations," Physics Letters A, vol. 364, no. 1, pp. 1-6, 2007.

[18] R. Liu, "Some new results on explicit traveling wave solutions of $k(m, n)$ equation," Discrete and Continuous Dynamical Systems B, vol. 13, no. 3, pp. 633-646, 2010.

[19] M. Li, S. C. Lim, and S. Y. Chen, "Exact solution of impulse response to a class of fractional oscillators and its stability," Mathematical Problems in Engineering, vol. 2011, Article ID 657839, 9 pages, 2011.

[20] J.-B. Li, "Exact traveling wave solutions and dynamical behavior for the $(n+1)$-dimensional multiple sine-Gordon equation," Science in China A, vol. 50, no. 2, pp. 153-164, 2007.

[21] C. Cattani and G. Pierro, "Complexity on acute myeloid leukemia mRNA transcript variant," Mathematical Problems in Engineering, vol. 2011, Article ID 379873, 16 pages, 2011.

[22] C. Cattani and A. Kudreyko, "Application of periodized harmonic wavelets towards solution of eigenvalue problems for integral equations," Mathematical Problems in Engineering, vol. 2010, Article ID 570136, 8 pages, 2010.

[23] Z. R. Liu and Z. Y. Ouyang, "A note on solitary waves for modified forms of Camassa-Holm and Degasperis-Procesi equations," Physics Letters A, vol. 366, no. 4-5, pp. 377-381, 2007.

[24] E. G. Bakhoum and C. Toma, "Specific mathematical aspects of dynamics generated by coherence functions," Mathematical Problems in Engineering, vol. 2011, Article ID 436198, 10 pages, 2011.

[25] M.-Y. Tang and W.-L. Zhang, "Four types of bounded wave solutions of CH- $\gamma$ equation," Science in China A, vol. 50, no. 1, pp. 132-152, 2007.

[26] Q.-D. Wang and M.-Y. Tang, "New explicit periodic wave solutions and their limits for modified form of Camassa-Holm equation," Acta Mathematicae Applicatae Sinica, vol. 26, no. 3, pp. 513-524, 2010.

[27] E. G. Bakhoum and C. Toma, "Mathematical transform of traveling-wave equations and phase aspects of quantum interaction," Mathematical Problems in Engineering, vol. 2010, Article ID 695208, 15 pages, 2010.

[28] R. Liu, "Several new types of solitary wave solutions for the generalized Camassa-Holm-DegasperisProcesi equation," Communications on Pure and Applied Analysis, vol. 9, no. 1, pp. 77-90, 2010.

[29] M. Li, "Fractal time series-a tutorial review," Mathematical Problems in Engineering, vol. 2010, Article ID 157264, 26 pages, 2010.

[30] S. Y. Chen, H. Tong, Z. Wang, S. Liu, M. Li, and B. Zhang, “Improved generalized belief propagation for vision processing," Mathematical Problems in Engineering, vol. 2011, Article ID 416963, 12 pages, 2011. 


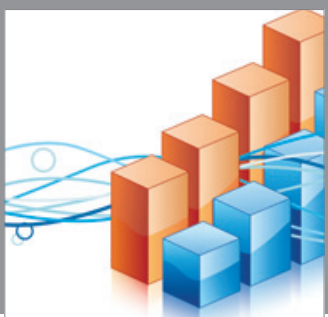

Advances in

Operations Research

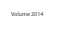

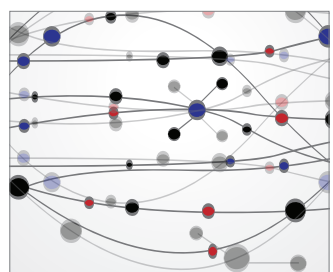

\section{The Scientific} World Journal
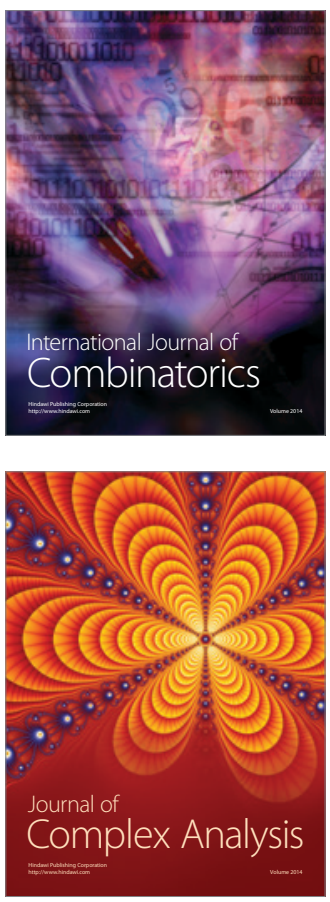

International Journal of

Mathematics and

Mathematical

Sciences
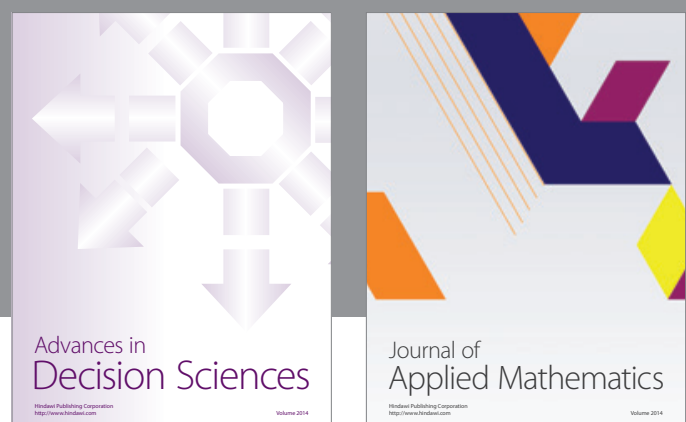

Journal of

Applied Mathematics
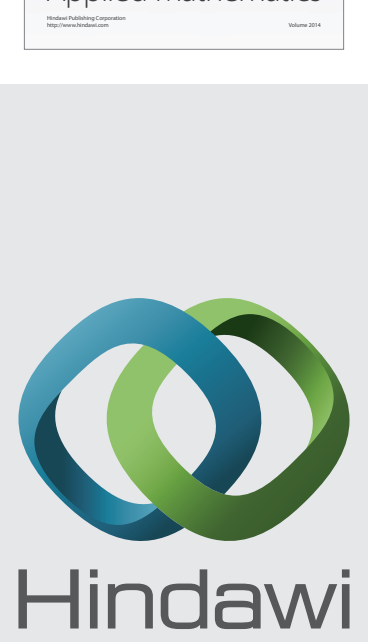

Submit your manuscripts at http://www.hindawi.com
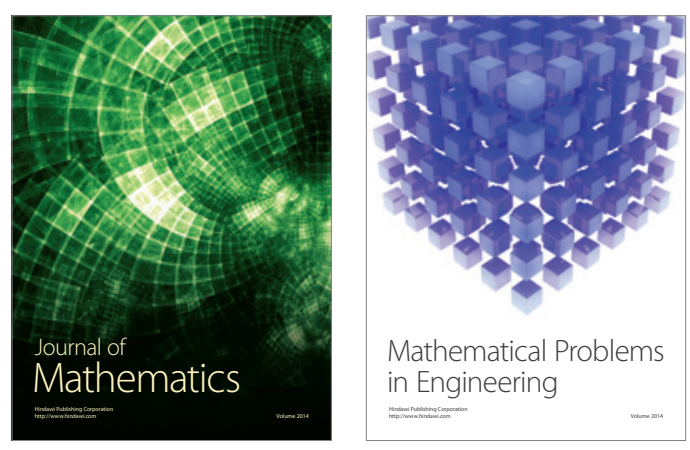

Mathematical Problems in Engineering
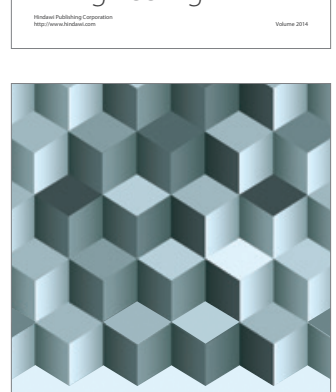

Journal of

Function Spaces
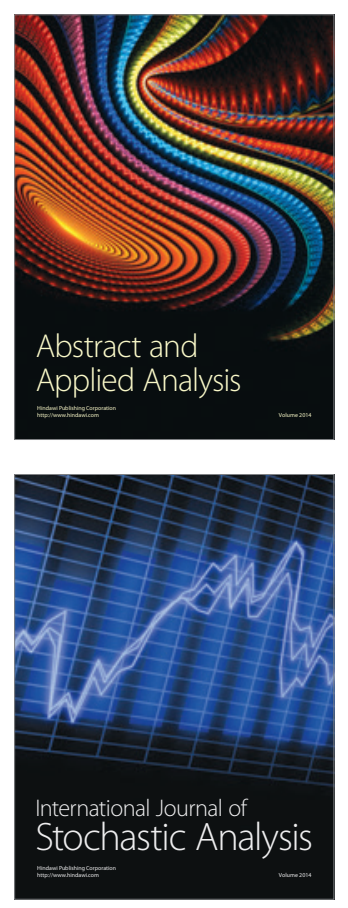

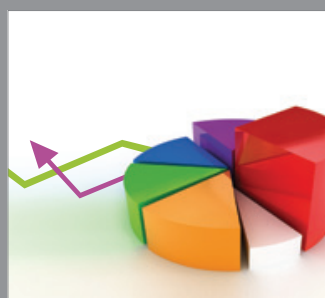

ournal of

Probability and Statistics

Promensencen
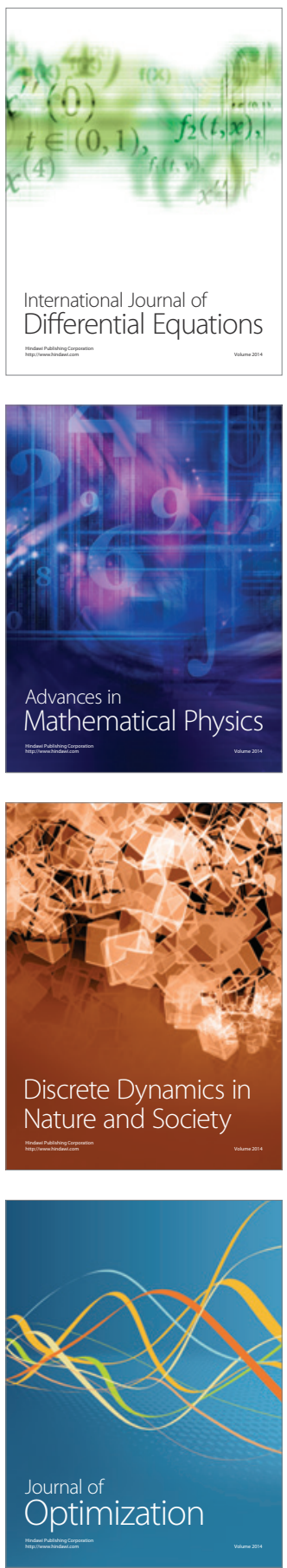